\title{
Higher Mortality in Bacteremic Sepsis - A Propensity Score Matched Study
}

\author{
Lisa Mellhammar ( $\square$ lisa.mellhammar@med.lu.se) \\ Lunds universitet Medicinska fakulteten https://orcid.org/0000-0002-7623-0342 \\ Fredrik Kahn \\ Lunds universitet Medicinska fakulteten \\ Caroline Whitlow \\ Lunds universitet Medicinska fakulteten \\ Thomas Kander \\ Lunds universitet Medicinska fakulteten \\ Bertil Christensson \\ Lunds universitet Medicinska fakulteten \\ Adam Linder \\ Lunds universitet Medicinska fakulteten
}

Research

Keywords: sepsis, bacteremia, antimicrobial therapy, mortality, organ dysfunction, propensity analysis, latent class analysis

Posted Date: December 14th, 2020

DOI: https://doi.org/10.21203/rs.3.rs-113804/v3

License: () (7) This work is licensed under a Creative Commons Attribution 4.0 International License. Read Full License

Version of Record: A version of this preprint was published at Scientific Reports on March 26th, 2021. See the published version at https://doi.org/10.1038/s41598-021-86346-4. 


\section{Abstract}

\section{Background}

Sepsis is a highly heterogenous disease which needs to be thoroughly mapped. The aim of this study was to describe characteristics and outcome for critically ill patients with sepsis-3 with either culture-positive or -negative sepsis.

Methods

Patients with severe sepsis or septic shock were retrospectively identified in the local quality registry from a general mixed Intensive Care Unit (ICU) at a University Hospital in 2007-2014. Data were collected through manual review of medical charts. Patients were included if they fulfilled sepsis-3 criteria and at least one blood culture was sampled $\pm 48 \mathrm{~h}$ from ICU admission. In a propensity score analysis bacteremic and non-bacteremic patients were matched 1:1 with regard to age, comorbidities, site of infection and antimicrobial therapy prior to blood cultures. A Latent Class Analysis (LCA) was performed to identify unmeasured class membership.

Results

784 patients were identified as treated in the ICU with a sepsis diagnosis. Blood cultures were missing in 140 excluded patients and additionally 95 patients did not fulfill a sepsis diagnosis and were also excluded. In total 549 patients were included, 295 (54\%) with bacteremia, 90 (16\%) were non-bacteremic but had relevant pathogens detected from another body location and in 164 (30\%) no relevant pathogen was detected in microbial samples. After the propensity score analysis ( $n=172$ in each group) 90-day mortality was higher in bacteremic patients, $47 \%$, than in non-bacteremic patients, $36 \%, p=0.04$.

Patients without antibiotic treatment before sample collection ( $n=352)$ were more often bacteremic, $63 \%$. Non-bacteremic patients without prior antibiotic treatment had lower mortality, 31\% ( $n=129)$, compared to non-bacteremic patients with prior antibiotic treatment, $51 \%$ $(\mathrm{n}=124), p<0.01$.

The LCA identified 8 classes, with different mortality rates, where pathogen detection in microbial sampleswere important factors for class distinction andoutcome.

\section{Conclusions}

Bacteremic patients had higher mortality than their non-bacteremic counter-parts. Bacteremia is more common in sepsis than previously reported, when studied in a clinical review. Clinical chart review should be considered gold standard since a significant proportion of the patients in the proposed sepsis cohort, did not have sepsis, but would have been included in ICD- or electronic health record (EHR) algorithm approaches.

\section{Background}

Sepsis is considered a "life-threatening organ dysfunction caused by a dysregulated host response to infection" and detailed data on its cause is fundamental (1).

Culture sampling is reported to only identify the causative organism in blood culture isolates in $15-30 \%$ of the sepsis patients (2-7). Additionally, $20-30 \%$ have a positive isolate from other locations. This means that at least $40 \%$ of sepsis patients are culture-negative or "sterile" $(2,6,8,9)$. The origin of this high rate of sterility is unknown. The low sensitivity of microbiological tests is believed to be one reason. Another factor to be considered is if antibiotics have been administered prior to collection of microbiological cultures. Other possible causes are viral infections, intermittent presence of bacteremia and non-infectious diagnoses misinterpreted as sepsis. The only treatments in sepsis are either antimicrobial therapy or supportive care. In sterile sepsis, the treatment remains empiric with risk to be ineffective. There is no effective treatment addressing the sepsis reaction, possibly due to the heterogeneity in sepsis. An endotype or subphenotype is a subset of a patient population defined by observable characteristics, distinguished from the population as a whole by natural history, disease manifestation and/or response to treatment.

Few studies specifically focus on the comparison of culture-negative and culture-positive sepsis or include culture status in identification of subphenotypes $(2,6,9-16)$.

The aim of this study was to describe characteristics and outcome for ICU patients with sepsis-3 with bacteremia, pathogen-detected but non-bacteremia, and for sterile sepsis, while taking treatment with antibiotics at the time of culture sampling into account as a confounder. 
We also aimed to identify subphenotypes of sepsis with culture status included as candidate clinical variables.

\section{Methods}

The primary outcome is whether there are differences in mortality between bacteremic sepsis and non-bacteremic sepsis.

Secondary outcomes are differences between the groups with regard to severity of disease, site of infection, comorbidities, preceding antibiotic therapy and other immunomodulatory medications and if there are subphenotypes with clinically distinction on prognosis.

\section{Patient selection and data collection}

Patients > 18 years admitted to the general intensive care unit (ICU) at Skåne University Hospital in Lund Sweden, between 2007 to 2014 and classified with the diagnosis sepsis or septic shock diagnosis during the length of stay, were eligible for inclusion. Additionally, medical charts of all patients were reviewed by an infectious disease specialist (LM) for accuracy of diagnosis and all patients without infection diagnosis as a cause for organ dysfunction according to sepsis-3 ( $\geq \Delta 2$ SOFA score) were excluded $(1,16)$. The Definitions of Infection in the Intensive Care Unit of the International Sepsis Forum Consensus Conference, were applied with exception for demands on microbiological verification (16). Further, at least one blood culture sampled between 48 hour before and 48 hour after ICU admission for each subject was required. Patients previously submitted to another ICU were excluded, as were re-admissions.

Patients were identified from the local quality registry (PASIVA Otimo Data AB, Kalmar, Sweden) and screened for inclusion. Date of admittance to and discharge from the ICU, mortality status at discharge from the ICU, survival days post discharge, Sequential Organ Failure Assessment (SOFA) scores, data on mechanical ventilation, renal replacement therapy and administration of vasopressors were also obtained from the local quality registry.

All cultures and other microbiological tests sampled within the 96-hour time frame, further sampling before/ after 96 hours, antibiotic treatment within 48 hours before first culture, source of infection, oral steroids, chemotherapy and immunomodulatory medications within 6 months before admission to ICU and comorbidities according to Charlson Comorbidity Index (CCI) were obtained through manual extraction from medical records.

All microorganisms in samples were assessed for sepsis-causing pathogen, colonization or contamination by an infectious disease specialist (LM). The microbial analyses were performed at the Clinical Microbiology Laboratory as part of clinical practice. For blood culture the BacT/Alert was used.

Ethical approval was obtained from the regional ethical board (decision number 2015/285).

\section{Statistical analysis}

Patients were categorized into bacteremic sepsis, pathogen-detected but non-bacteremic sepsis, and sterile sepsis. Categorical variables are presented as frequencies and percentages and continuous variables as medians with 95\% Confidence Interval (Cl) and interquartile range (IQR). The groups were compared with Pearsons $\chi^{2}$ or Fisher's exact test for proportions, as appropriate, while Mann-Whitney $U$ test and Kruskal Wallis were used for comparison of medians.

We used logistic regression for propensity score matching to balance groups of bacteremic and non-bacteremic patients with potential confounders; age, Charlson comorbidity index, preceding antibiotic therapy and site of infection. We did not include scores for severity of disease in the primary analysis, as we see it as an intermediate in sepsis progression rather than a confounder. We used "nearestneighbour" matching without replacement at a ratio of 1:1 and accepted a propensity variable match tolerance of 0.01 . $\chi^{2}$ or Fishers exact test was used to compare baseline categorical variables and Mann-Whitney U test for continuous variables.

In sepsis, where a formal definition is a construction, subphenotypes can be constructed out of observable characteristics. Latent Class Analysis (LCA), a machine-learning approach, is a statistical method to identify unmeasured class membership in order to derive subphenotypes in a non-biased way. In contrast to traditional regression analysis which finds association between variables and a known outcome, the LCA models create classes defined by variables without consideration of the outcome.

LCA was used to identify sepsis classes. The number of classes were tested for model fit, starting from one until additional classes did not improve the model fit. The model fit was estimated by Akaike Information Criterion (AIC), Bayesian Information Criterion (BIC), adjusted Bayesian Information Criterion (ABIC) and bootstrapped likelihood ratio test. The classes obtained were also inspected for clinical meaningfulness. 
90-day mortality was compared by the log rank test.

Analyses were performed using SPSS software system version 24.0 (IBM, Armonk, NY), with the FUZZY extension except for LCA analyses, which were conducted using Mplus (version 8.14; Muthén \& Muthén, Los Angeles, CA), with data preparation in R version 3.4 .0 (The R Foundation for Statistical Computing).

\section{Results}

\section{Patient characteristics}

A flow chart of patients is presented in Fig. 1. Over the eight study years, 784 patients were treated in the ICU and received a sepsis or septic shock diagnosis. Microbiological samples were not obtained within the predefined time interval in 88 excluded patients. Additionally 52 , lacked blood cultures and were excluded. Ninety-five patients were excluded at the medical chart review due to absence of sepsis-3 diagnosis and 549 patients were finally included in the study. Blood cultures were positive in 286 of the patients, 83 had negative blood cultures but a positive culture from another body location classified as the focus of infection. One hundred and eighty patients were sterile without positive cultures. Four sterile patients were positive in polymerase chain reaction assay (PCR); 2 were positive for legionella and 1 for influenza in respiratory tract samples and 1 for meningococci in cerebrospinal fluid and were reclassified as pathogen-detected but nonbacteremic sepsis. Additionally, nine patients with positive blood culture and 3 patients where pathogens were detected from the site of infection, outside the 96-hour time frame surrounding ICU admission, were reclassified as bacteremic and pathogen-detected but nonbacteremic, respectively. This resulted in $54 \%(n=295)$ bacteremic sepsis, $16 \%(n=90)$ pathogen-detected but non-bacteremic sepsis and $30 \%(n=164)$ sterile sepsis, see Fig. 1.

There were no missing data in the primary analyses. There were no differences in mortality between the groups of different microbiological status, Fig. 2a. For differences in demography and clinical characteristics between bacteremic and non-bacteremic patients see Table 1and Table 2. 
Table 1

Demography and morbidity

\begin{tabular}{|c|c|c|c|c|c|}
\hline & \multirow[t]{3}{*}{$\begin{array}{l}\text { Bacteremic } n= \\
295\end{array}$} & \multirow[t]{3}{*}{$\begin{array}{l}\text { Pathogen detected, non-bacteremic } n \\
=90\end{array}$} & \multirow[t]{3}{*}{$\begin{array}{l}\text { Sterile } \mathrm{n}= \\
164\end{array}$} & & \multirow{3}{*}{$\begin{array}{l}\text { bacteremic vs not } \\
\text { p }\end{array}$} \\
\hline & & & & \multirow[b]{2}{*}{$p$} & \\
\hline & & & & & \\
\hline Age, median & $67(59-75)$ & $66(55-73)$ & $67(58-75)$ & 0.47 & 0.37 \\
\hline Gender, female & $127(43)$ & $42(47)$ & $71(43)$ & 0.83 & 0.74 \\
\hline \multicolumn{6}{|l|}{ Comorbidities } \\
\hline Charlson $\mathrm{Cl}$, median & $2(2-3)$ & $2(0-3)$ & $2(1-2)$ & 0.97 & 0.82 \\
\hline Liver disease & $16(5)$ & $1(1)$ & $2(1)$ & 0.03 & $<0.01$ \\
\hline Renal disease & $27(9)$ & $4(4)$ & $11(7)$ & 0.29 & 0.15 \\
\hline Diabetes mellitus & $62(21)$ & $16(18)$ & $34(21)$ & 0.82 & 0.77 \\
\hline Acute myocardial infarction & $38(13)$ & $18(20)$ & $28(17)$ & 0.19 & 0.09 \\
\hline Congestive heart failure & $34(12)$ & $8(9)$ & $18(11)$ & 0.78 & 0.64 \\
\hline Peripheral vascular disease & $10(3)$ & $6(7)$ & $5(3)$ & 0.31 & 0.56 \\
\hline Cerebrovascular accident & $20(7)$ & $9(10)$ & $14(9)$ & 0.56 & 0.32 \\
\hline Hemiplegia & $9(3)$ & $3(3)$ & $6(4)$ & 0.94 & 0.74 \\
\hline Dementia & $3(1)$ & $1(1)$ & $4(3)$ & 0.45 & 0.48 \\
\hline COPD & $26(9)$ & $11(12)$ & $22(14)$ & 0.27 & 0.11 \\
\hline Connective tissue disease & $23(8)$ & $9(10)$ & $12(7)$ & 0.74 & 0.83 \\
\hline Peptic ulcer disease & $13(4)$ & $4(4)$ & $5(3)$ & 0.76 & 0.61 \\
\hline Malignancy & $88(30)$ & $25(28)$ & $48(29)$ & 0.93 & 0.78 \\
\hline \multicolumn{6}{|l|}{ Medication } \\
\hline Oral steroids & $40(14)$ & $17(19)$ & $25(15)$ & 0.46 & 0.32 \\
\hline Chemotherapy & $48(16)$ & $13(14)$ & $19(12)$ & 0.41 & 0.23 \\
\hline $\begin{array}{l}\text { Immunomodulatory } \\
\text { medication }\end{array}$ & $13(4)$ & $7(8)$ & $14(9)$ & 0.16 & 0.06 \\
\hline Preceding antibiotic therapy & $72(24)$ & $40(44)$ & $84(52)$ & $\dot{0} 01$ & $<0.01$ \\
\hline \multicolumn{6}{|l|}{ Severity of disease } \\
\hline SOFA max, median & $11(9-14)$ & $9(8-12)$ & $10(7-13)$ & $\dot{0} 01$ & $<0.01$ \\
\hline SOFA resp max, median & $3(2-4)$ & $3(3-4)$ & $3(3-4)$ & 0.32 & 0.14 \\
\hline SOFA renal max, median & $2(1-4)$ & $1(0-2)$ & $1(0-3)$ & $\dot{0} 01$ & $<0.01$ \\
\hline SOFA liver max, median & $0(0-2)$ & $0(0-1)$ & $0(0-1)$ & 0.04 & 0.02 \\
\hline SOFA coag max, median & $2(0-3)$ & $2(0-3)$ & $1(1-2)$ & $\dot{0} 01$ & $<0.01$ \\
\hline
\end{tabular}

Percent within parenthesis for proportions, interquartile range within parenthesis for medians

$\mathrm{Cl}=$ Comorbidity Index, $\mathrm{COPD}=$ Chronic Obstructive Pulmonary Disease, SOFA = Sequential Organ Failure Assessment, $\mathrm{CNS}=$ Central Nervous System 


\begin{tabular}{|c|c|c|c|c|c|}
\hline & \multirow{3}{*}{$\begin{array}{l}\text { Bacteremic } n= \\
295\end{array}$} & \multirow{3}{*}{$\begin{array}{l}\text { Pathogen detected, non-bacteremic } n \\
=90\end{array}$} & \multirow{3}{*}{$\begin{array}{l}\text { Sterile } n= \\
164\end{array}$} & & \multirow{3}{*}{$\begin{array}{l}\text { bacteremic vs no } \\
\text { p }\end{array}$} \\
\hline & & & & \multirow[b]{2}{*}{$\mathbf{p}$} & \\
\hline & & & & & \\
\hline $\begin{array}{l}\text { SOFA cardiovasc max, } \\
\text { median }\end{array}$ & $3(1-4)$ & $3(1-4)$ & $3(1-4)$ & 0.66 & 0.38 \\
\hline SOFA CNS max, median & $1(0-2)$ & $1(0-2)$ & $1(0-2)$ & 0.88 & 0.85 \\
\hline $\begin{array}{l}\text { Lactate max mmol/L, } \\
\text { median }\end{array}$ & $4.4(2.8-7.2)$ & $4.0(2.6-6.5)$ & $\begin{array}{l}3.6(2.4- \\
5.8)\end{array}$ & 0.01 & 0.01 \\
\hline \multicolumn{6}{|l|}{ Outcome } \\
\hline ICU mortality & $71(24)$ & $13(14)$ & $46(28)$ & 0.05 & 0.82 \\
\hline 90-day mortality & $125(42)$ & $32(36)$ & $72(44)$ & 0.41 & 0.74 \\
\hline \multicolumn{6}{|c|}{ Percent within parenthesis for proportions, interquartile range within parenthesis for medians } \\
\hline
\end{tabular}


Table 2

Infection, microbial findings and cultures

\begin{tabular}{|c|c|c|c|c|c|}
\hline & $\begin{array}{l}\text { Bacteremic } n= \\
295\end{array}$ & $\begin{array}{l}\text { Pathogen detected non-bacteremic } n= \\
90\end{array}$ & $\begin{array}{l}\text { Sterile } \mathrm{n}= \\
164\end{array}$ & $p$ & $\begin{array}{l}\text { bacteremic vs not } \\
p\end{array}$ \\
\hline \multicolumn{6}{|l|}{ Foci } \\
\hline Respiratory tract & $49(17)$ & $27(30)$ & $57(35)$ & $\dot{0} 01$ & $<0.01$ \\
\hline Urinary tract & $66(23)$ & $7(8)$ & $4(2)$ & $<01$ & $<0.01$ \\
\hline Abdominal & $41(14)$ & $21(23)$ & $52(32)$ & $<.01$ & $<0.01$ \\
\hline Postoperative & $17(6)$ & $13(14)$ & $20(12)$ & 0.01 & $<0.01$ \\
\hline Central nervous system & $11(4)$ & $2(2)$ & $1(1)$ & 0.12 & 0.06 \\
\hline Bone \& joint & $7(2)$ & $2(2)$ & $2(1)$ & 0.69 & 0.51 \\
\hline Heart & $10(3)$ & $2(2)$ & $3(2)$ & 0.59 & 0.43 \\
\hline Skin \& soft tissue & $30(10)$ & $12(13)$ & $5(3)$ & $\dot{0} 01$ & 0.15 \\
\hline Other or unknown & $64(22)$ & $2(2)$ & $20(12)$ & $\dot{0} 01$ & $<0.01$ \\
\hline Nosocomial & $30(10)$ & $18(20)$ & $36(22)$ & 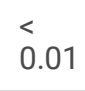 & $<0.01$ \\
\hline \multicolumn{6}{|l|}{ Pathogens } \\
\hline E. coli & $78(26)$ & $10(11)$ & & $<.01$ & \\
\hline S. aureus & $30(10)$ & $7(8)$ & & 0.50 & \\
\hline S. pneumoniae & $34(12)$ & $10(11)$ & & 0.91 & \\
\hline Other streptococci & $38(13)$ & $16(18)$ & & 0.24 & \\
\hline Other enterobacterales & $26(9)$ & $6(7)$ & & 0.52 & \\
\hline Enterococci & $12(4)$ & $2(2)$ & & 0.54 & \\
\hline Meningococci & $3(1)$ & $1(1)$ & & 1.0 & \\
\hline Candida & $8(3)$ & $1(1)$ & & 0.69 & \\
\hline P. aeruginosa & $11(4)$ & $2(2)$ & & 0.74 & \\
\hline Other & $55(19)$ & $35(39)$ & & $<.01$ & \\
\hline \multicolumn{6}{|c|}{ No of patients with cultures obtained } \\
\hline \multicolumn{6}{|l|}{ Blood culture pairs } \\
\hline 1 & $295(100)$ & $90(100)$ & $164(100)$ & 1.0 & 1.0 \\
\hline 2 & $258(87)$ & $58(64)$ & $108(66)$ & $<.01$ & $<0.01$ \\
\hline 3 & $82(28)$ & $20(22)$ & $36(22)$ & 0.30 & 0.12 \\
\hline 4 & $18(6)$ & $5(6)$ & $10(6)$ & 0.96 & 0.92 \\
\hline $\begin{array}{l}\text { Blood culture pairs, } \\
\text { mean }\end{array}$ & $2.2(2.1-2.3)$ & $1.9(1.8-2.0)$ & $1.9(1.8-2.0)$ & $<01$ & $<0.01$ \\
\hline
\end{tabular}

Percent within parenthesis for proportions, $95 \%$ confidence interval within parenthesis for means 


\begin{tabular}{|c|c|c|c|c|c|}
\hline & $\begin{array}{l}\text { Bacteremic } n= \\
295\end{array}$ & $\begin{array}{l}\text { Pathogen detected non-bacteremic } n= \\
90\end{array}$ & $\begin{array}{l}\text { Sterile } n= \\
164\end{array}$ & $p$ & $\begin{array}{l}\text { bacteremic vs not } \\
p\end{array}$ \\
\hline Respiratory tract & $82(28)$ & $38(42)$ & $68(41)$ & $<0.01$ & $<0.01$ \\
\hline Feces & $33(11)$ & $11(12)$ & $11(7)$ & 0.05 & 0.23 \\
\hline Urine & $228(77)$ & $64(71)$ & $108(66)$ & $\begin{array}{l}< \\
0.01\end{array}$ & 0.03 \\
\hline Wound & $88(30)$ & $41(46)$ & $28(17)$ & $<.01$ & 0.98 \\
\hline Drainage & $8(3)$ & $10(11)$ & $9(5)$ & 0.02 & 0.02 \\
\hline Synovial fluid & $3(1)$ & $3(3)$ & $0(0)$ & $\begin{array}{l}< \\
0.04\end{array}$ & 1.0 \\
\hline Cerebrospinal fluid & $20(7)$ & $3(3)$ & $5(3)$ & 0.19 & $<0.01$ \\
\hline \multicolumn{6}{|c|}{ No of patients with cultures from presumed infection foci } \\
\hline Respiratory tract & $36(73)$ & $25(93)$ & $41(72)$ & 0.09 & 0.50 \\
\hline Urine & $61(92)$ & $7(100)$ & $3(75)$ & 0.33 & 0.86 \\
\hline Postoperative & $11(65)$ & $12(92)$ & $11(52)$ & 0.08 & 0.94 \\
\hline Central nervous system & $11(100)$ & $2(100)$ & $1(100)$ & 1.0 & 1.0 \\
\hline Bone \& joint & $4(57)$ & $1(50)$ & $2(100)$ & 0.97 & 0.55 \\
\hline
\end{tabular}

\section{Patients without preceding antibiotic therapy}

In 352 patients not treated with antibiotics prior to culture sampling, 63\% $(n=223)$ were bacteremic, $14 \%(n=50)$ were non-bacteremic but with positive microbial samples from foci of infection, and $22 \%(n=79)$ were sterile sepsis.

These patients had similar characteristics except for renal disease being more common among bacteremic patients than non-bacteremic patients, 20 versus 4 patients, respectively, $p=0.04$. Chronic obstructive pulmonary disease (COPD) was, however, less common among bacteremic patients, 20 patients versus 21 patients, $p=0.04$, and likewise immunomodulatory medication in 6 bacteremic patients versus 10 non-bacteremic patients, $p=0.03$.

\section{Microbial samples}

Escherichia coli was the most common pathogen detected from blood, and non-pneumococcal streptococci were the most common pathogens from other non-blood sites.

Patients from the bacteremic sepsis, pathogen-detected but non-bacteremic sepsis or sterile sepsis groups were compared for proportion of patients with microbial samples from presumed infectious foci. Generally, more microbial samples were sampled from patients with pathogens detected. When compared for proportion of patients with microbial samples from presumed infection foci, the differences between pathogen-detected and sterile sepsis did not remain for respiratory tract, urinary tract, central nervous system, bone and joints and drainage or wound for postoperative infections. A higher number of blood culture pairs were drawn in bacteremic patients, (mean $2.2,95 \%$ $\mathrm{Cl}$ 2.1-2.2) compared to non-bacteremic patients (mean 1.9, 95\% $\mathrm{Cl} 1.8-2.0) p<0.01$. All patients had at least one pair of blood culture drawn. Bacteremic patients had a higher proportion of a second pair of blood cultures drawn. The additional rate of positivity in the second pair of blood culture was $7.4 \%$ for the bacteremic patients. The addition of a second pair of blood culture in the non-bacteremic patients with only one pair of blood culture drawn, with an equal rate of positivity as in the bacteremic sepsis, would be equivalent to 4 more patients becoming bacteremic (Table 2).

\section{Outcome}


As comparisons of mortality between groups of patients with positive respectively negative in microbiological samples are most likely vulnerable to confounders, a propensity score analysis was performed. There were no missing data on the variables in the propensity score analysis.

In the entire cohort 172 matched pairs were retrieved, for clinical characteristics in the matched groups, see Table 3. Mortalities were significantly higher among bacteremic patients as were the total SOFA score, the SOFA scores for renal function, liver function, coagulation and lactate, Fig. 2b. 
Table 3

Propensity matched cohort

\begin{tabular}{|c|c|c|c|}
\hline & \multicolumn{2}{|c|}{ Propensity matched cohort } & \\
\hline & \multirow[t]{2}{*}{ Bacteremic $n=172$} & \multirow[t]{2}{*}{ Non bacteremic $n=172$} & \\
\hline & & & $\mathrm{p}$ \\
\hline Age, median & $67(59-75)$ & $66(55-74)$ & 0.42 \\
\hline Gender, female & $76(40)$ & $84(44)$ & 0.41 \\
\hline Charlson $\mathrm{Cl}$, median & $1(0-2)$ & $1(0-2)$ & 0.89 \\
\hline Liver disease & $4(2)$ & $3(2)$ & 0.74 \\
\hline Renal disease & $15(9)$ & $9(5)$ & 0.20 \\
\hline Diabetes mellitus & $34(20)$ & $36(21)$ & 0.79 \\
\hline Cardiovascular disease & $41(24)$ & $48(28)$ & 0.39 \\
\hline Cerebral disease & $17(10)$ & $20(12)$ & 0.60 \\
\hline COPD & $15(9)$ & $22(13)$ & 0.22 \\
\hline Connective tissue disease & $13(8)$ & $15(9)$ & 0.69 \\
\hline Peptic ulcer disease & $10(6)$ & $7(4)$ & 0.46 \\
\hline Malignancy & $45(26)$ & $46(27)$ & 0.90 \\
\hline Immunosuppressant & $46(27)$ & $40(23)$ & 0.46 \\
\hline Preceding antibiotic therapy & $60(35)$ & $59(34)$ & 0.91 \\
\hline \multicolumn{4}{|l|}{ Foci } \\
\hline Respiratory tract & $39(23)$ & $38(22)$ & 0.90 \\
\hline Urinary tract & $11(6)$ & $11(6)$ & 1.0 \\
\hline Abdominal & $38(22)$ & $40(23)$ & 0.80 \\
\hline Bone \& joint & $5(3)$ & $4(2)$ & 0.74 \\
\hline Skin \& soft tissue & $20(12)$ & $16(9)$ & 0.48 \\
\hline Other & $59(34)$ & $63(37)$ & 0.65 \\
\hline \multicolumn{4}{|l|}{ Outcome } \\
\hline ICU mortality & $49(29)$ & $37(22)$ & 0.14 \\
\hline 90-day mortality & $81(47)$ & $62(36)$ & 0.04 \\
\hline \multicolumn{4}{|l|}{ Severity of disease } \\
\hline SOFA max, median & $12(9-16)$ & $10(7-13)$ & $<0.01$ \\
\hline SOFA resp max, median & $3(2-4)$ & $3(3-4)$ & 0.33 \\
\hline SOFA renal max, median & $2(0-4)$ & $1(0-3)$ & 0.02 \\
\hline SOFA liver max, median & $0(0-2)$ & $0(0-1)$ & 0.05 \\
\hline SOFA coag max, median & $2(0-3)$ & $1(0-2)$ & $<0.01$ \\
\hline SOFA cardiovasc max, median & $3(1-4)$ & $3(1-4)$ & 0.58 \\
\hline
\end{tabular}

Percent within parenthesis for proportions, interquartile range within parenthesis for medians

$\mathrm{Cl}=$ Comorbidity Index, $\mathrm{COPD}=$ Chronic Obstructive Pulmonary Disease, SOFA = Sequential Organ Failure Assessment, $\mathrm{CNS}=$ Central Nervous System 


\section{Propensity matched cohort}

$\begin{array}{llll}\text { SOFA CNS max, median } & 1(0-3) & 1(0-2) & 0.28 \\ \text { Lactate max mmol/L, median } & 4.4(3.0-8.0) & 3.8(2.4-6.5)\end{array}$

Percent within parenthesis for proportions, interquartile range within parenthesis for medians

$\mathrm{Cl}=$ Comorbidity Index, $\mathrm{COPD}=$ Chronic Obstructive Pulmonary Disease, $\mathrm{SOFA}=$ Sequential Organ Failure Assessment, $\mathrm{CNS}=\mathrm{Central}$ Nervous System

Since the matching affected the mortality, the mortality was also compared for patients who had received antibiotic therapy prior to blood cultures. Non-bacteremic patients had significantly higher mortality if they received antibiotic therapy prior to blood cultures than if they had not received antibiotic therapy, Fig. 2c.

\section{Latent Class Analysis}

The following baseline variables were categorized and entered into a LCA-model: gender, bacteremia, pathogen-detected but non-bacteremic or sterile sepsis, sepsis-causing pathogen, foci of infection, prior antibiotic treatment, immunomodulatory medications, nosocomial infection, fever $\left(>38^{\circ} \mathrm{C}\right)$, hypothermia $\left(<36^{\circ} \mathrm{C}\right)$, acidosis $(\mathrm{pH}<7.35)$, p-lactate (divided in quartiles; 0-1.8, > 1.8-3,>3-5.1,>5.1), and CNS-, respiratory-, cardiovascular-, renal-, liver- or coagulatory organ dysfunction. 469 patients without any missing data on the baseline variables were entered into the LCA. An eight-class model or a nine-class model were the best fit for the cohort, ABIC continued to decrease until 9 classes model, Table 4. We inspected the 8- and 9-class models and the 8-class model did yield more clinically meaningful classes and hence was chosen. The representation of class membership for baseline variables are presented in Table 5 . The eight classes are characterized by:

Table 4

LCA models' fit

\begin{tabular}{|c|c|c|c|c|c|c|c|c|}
\hline & $\begin{array}{l}\text { Loglikelehood } \\
\text { HO value }\end{array}$ & AIC & BIC & $A B I C$ & Entropy & $\begin{array}{l}\text { Vouong-Lo- } \\
\text { Mendel-Rubin } \\
p\end{array}$ & $\begin{array}{l}\text { Adjusted Lo- } \\
\text { Mendel-Rubin } \\
p\end{array}$ & $\begin{array}{l}\text { Bootstraped } \\
\text { likelood ratic } \\
\text { test } p\end{array}$ \\
\hline 1-class & -5861.017 & 11776.033 & 11888.100 & 11802.407 & & & & \\
\hline $\begin{array}{l}2- \\
\text { classes }\end{array}$ & -5414.615 & 10939.231 & 11167.514 & 10992.955 & 0.989 & $<0.0001$ & $<0.0001$ & $<0.0001$ \\
\hline $\begin{array}{l}\text { 3- } \\
\text { classes }\end{array}$ & -5299.530 & 10765.000 & 11109.560 & 10846.134 & 0.943 & $<0.0001$ & $<0.0001$ & $<0.0001$ \\
\hline $\begin{array}{l}\text { 4- } \\
\text { classes }\end{array}$ & -5217.986 & 10657.972 & 11118.689 & 10766.397 & 0.957 & 0.6171 & 0.6198 & $<0.0001$ \\
\hline $\begin{array}{l}5- \\
\text { classes }\end{array}$ & -5161.312 & 10600.623 & 11177.557 & 10736.399 & 0.948 & 0.77 & 0.77 & $<0.0001$ \\
\hline $\begin{array}{l}\text { 6- } \\
\text { classes }\end{array}$ & -5103.545 & 10541.090 & 11234.241 & 10704.216 & 0.956 & 0.76 & 0.76 & $<0.0001$ \\
\hline $\begin{array}{l}\text { 7- } \\
\text { classes }\end{array}$ & -5052.411 & 10494.822 & 11304.190 & 10685.299 & 0.96 & 0.76 & 0.76 & $<0.0001$ \\
\hline $\begin{array}{l}\text { 8- } \\
\text { classes }\end{array}$ & -5001.904 & 10449.809 & 11375.393 & 10667.636 & 0.943 & 0.77 & 0.77 & $<0.0001$ \\
\hline $\begin{array}{l}\text { 9- } \\
\text { classes }\end{array}$ & -4958.769 & 10419.538 & 11461.339 & 10664.716 & 0.923 & 0.86 & 0.86 & $<0.0001$ \\
\hline $\begin{array}{l}10- \\
\text { classes }\end{array}$ & -4924.905 & 10407.810 & 11565.828 & 10680.338 & 0.927 & 0.81 & 0.81 & - \\
\hline
\end{tabular}


Table 5

Selected baseline variables in the LCA

\begin{tabular}{|c|c|c|c|c|c|c|c|c|c|}
\hline & Class 1 & Class 2 & Class 3 & Class 4 & Class 5 & Class 6 & Class 7 & Class 8 & \\
\hline & $\begin{array}{l}\text { Nosocomial } \\
\text { sterile } \\
\text { sepsis }\end{array}$ & $\begin{array}{l}\text { Sepsis } \\
\text { with } \\
\text { lactic } \\
\text { acidosis }\end{array}$ & $\begin{array}{l}\text { Community- } \\
\text { acquired } \\
\text { sterile }\end{array}$ & $\begin{array}{l}\text { Abdominal } \\
\text { pathogen } \\
\text { detected }\end{array}$ & $\begin{array}{l}\text { Nosocomial } \\
\text { pathogen } \\
\text { detected }\end{array}$ & $\begin{array}{l}\text { Gram- } \\
\text { positive } \\
\text { bacteria }\end{array}$ & $\begin{array}{l}\text { Immuno- } \\
\text { suppressed } \\
\text { sepsis }\end{array}$ & $\begin{array}{l}\text { Urinary } \\
\text { tract } \\
\text { infection } \\
\text { sepsis }\end{array}$ & $p$ \\
\hline & $n=20$ & $\mathrm{n}=37$ & $n=130$ & $n=56$ & $\mathrm{n}=34$ & $\mathrm{n}=112$ & $n=20$ & $n=60$ & \\
\hline Gender, female & $10(10)$ & $15(41)$ & $54(42)$ & $26(46)$ & $12(35)$ & $47(42)$ & $5(25)$ & $30(50)$ & 0.59 \\
\hline Bacteremia & $0(0)$ & $34(92)$ & $0(0)$ & $28(50)$ & $22(65)$ & $86(77)$ & $20(100)$ & $58(97)$ & $\begin{array}{l}< \\
0.01\end{array}$ \\
\hline $\begin{array}{l}\text { Pathogen- } \\
\text { detected }\end{array}$ & $0(0)$ & $\begin{array}{l}37 \\
(100)\end{array}$ & $5(4)$ & $56(100)$ & $34(100)$ & $\begin{array}{l}112 \\
(100)\end{array}$ & $20(100)$ & $60(100)$ & $<0.01$ \\
\hline \multicolumn{10}{|l|}{ Foci of infection } \\
\hline Respiratory tract & $0(0)$ & $4(11)$ & $56(43)$ & 8 (14) & $0(0)$ & $45(40)$ & $0(0)$ & $2(3)$ & 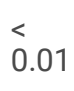 \\
\hline Urinary tract & $0(0)$ & $4(11)$ & $4(3)$ & $3(5)$ & $3(9)$ & $0(0)$ & $0(0)$ & $50(83)$ & $\begin{array}{l}< \\
0.01\end{array}$ \\
\hline Abdominal & $0(0)$ & $14(38)$ & $49(38)$ & $34(61)$ & $0(0)$ & $0(0)$ & $0(0)$ & $4(7)$ & $<0.01$ \\
\hline Postoperative & $20(100)$ & $0(0)$ & $0(0)$ & $0(0)$ & $28(82)$ & $0(0)$ & $0(0)$ & $0(0)$ & $\begin{array}{l}< \\
0.01\end{array}$ \\
\hline $\begin{array}{l}\text { Skin \& soft } \\
\text { tissue }\end{array}$ & $0(0)$ & $0(0)$ & $2(2)$ & $8(14)$ & $0(0)$ & $27(24)$ & $1(5)$ & $0(0)$ & $<.01$ \\
\hline Nosocomial & $20(100)$ & $3(8)$ & $12(9)$ & $2(4)$ & $34(100)$ & $0(0)$ & $1(5)$ & $0(0)$ & $\begin{array}{l}< \\
0.01\end{array}$ \\
\hline $\begin{array}{l}\text { Preceding } \\
\text { antibiotics }\end{array}$ & $12(60)$ & $9(24)$ & $65(50)$ & $25(10)$ & $10(29)$ & $23(21)$ & $19(95)$ & $4(7)$ & $<0.01$ \\
\hline $\begin{array}{l}\text { Immuno- } \\
\text { suppression }\end{array}$ & $2(10)$ & $11(30)$ & $33(25)$ & $18(32)$ & $5(15)$ & $13(12)$ & $17(85)$ & $4(7)$ & $\begin{array}{l}< \\
0.01\end{array}$ \\
\hline \multicolumn{10}{|l|}{$\begin{array}{l}\text { Organ } \\
\text { dysfunction }\end{array}$} \\
\hline Respiratory & $15(73)$ & $\begin{array}{l}37 \\
(100)\end{array}$ & $128(98)$ & $51(92)$ & $34(100)$ & $\begin{array}{l}101 \\
(90)\end{array}$ & $18(90)$ & $54(89)$ & 0.01 \\
\hline Coagulopathy & $1(5)$ & $26(70)$ & $36(28)$ & $3(5)$ & $6(18)$ & $43(38)$ & $19(95)$ & $31(53)$ & $\begin{array}{l}< \\
0.01\end{array}$ \\
\hline Liver & $2(10)$ & $22(59)$ & $16(12)$ & $3(5)$ & $3(9)$ & $13(12)$ & $5(25)$ & $8(13)$ & $<01$ \\
\hline Cardiovascular & $16(80)$ & $35(96)$ & $81(62)$ & $31(55)$ & $13(38)$ & $78(69)$ & $11(55)$ & $40(60)$ & 0.02 \\
\hline CNS & $6(30)$ & $22(59)$ & $40(31)$ & $13(10)$ & $0(0)$ & $44(39)$ & $0(0)$ & $15(2)$ & $<.01$ \\
\hline Acidosis & $7(35)$ & $35(95)$ & $64(49)$ & $21(38)$ & $14(41)$ & 66 (59) & $2(10)$ & $12(20)$ & $\begin{array}{l}< \\
0.01\end{array}$ \\
\hline Lactate $<1.7$ & $9(45)$ & $0(0)$ & $32(25)$ & $11(20)$ & $7(21)$ & $39(35)$ & $6(30)$ & $8(13)$ & $\begin{array}{l}< \\
0.01\end{array}$ \\
\hline Lactate 1.8-3 & $5(25)$ & $1(3)$ & $40(31)$ & $14(25)$ & $9(27)$ & $27(24)$ & $24(27)$ & $6(30)$ & 0.09 \\
\hline Lactate $3.1-5.1$ & $3(15)$ & $4(11)$ & $35(27)$ & $19(34)$ & $7(21)$ & $20(18)$ & $5(25)$ & $21(35)$ & 0.04 \\
\hline Lactate $>5.1$ & $3(15)$ & $32(87)$ & $23(18)$ & $12(21)$ & $11(32)$ & $26(23)$ & $3(15)$ & $15(25)$ & $\begin{array}{l}< \\
0.01\end{array}$ \\
\hline
\end{tabular}




\begin{tabular}{|llllllllll|}
\hline & Class 1 & Class 2 & Class 3 & Class 4 & Class 5 & Class 6 & Class 7 & Class 8 \\
\hline Fever & $8(40)$ & $4(11)$ & $36(28)$ & $10(18)$ & $10(29)$ & $41(37)$ & $7(35)$ & $14(23)$ & 0.04 \\
\hline Hypothermia & $1(5)$ & $12(32)$ & $20(15)$ & $5(9)$ & $2(6)$ & $10(9)$ & $1(5)$ & $5(8)$ & $<$ \\
\hline Pathogen & & & & & & & & \\
\hline S.aureus & $0(0)$ & $1(3)$ & $1(1)$ & $0(0)$ & $4(12)$ & $24(21)$ & $3(15)$ & $0(0)$ & $<$ \\
\hline Enterobacterales & $0(0)$ & $15(41)$ & $1(1)$ & $10(18)$ & $8(24)$ & $3(3)$ & $6(30)$ & $55(92)$ & $<$ \\
\hline Streptococci & $0(0)$ & $0(0)$ & $0(0)$ & $2(4)$ & $3(9)$ & $77(69)$ & $2(10)$ & $0(0)$ & $<.01$ \\
\hline Candida & $0(0)$ & $1(3)$ & $0(0)$ & $0(0)$ & $3(9)$ & $0(0)$ & $2(10)$ & $0(0)$ & $<$ \\
\hline Others & $0(0)$ & $20(54)$ & $2(2)$ & $44(79)$ & $16(47)$ & $8(7)$ & $7(35)$ & $5(5)$ & $<.01$ \\
\hline CNS = Central Nervous System & & & & & & & 0.01 \\
\hline
\end{tabular}

1. Nosocomial, sterile sepsis, in this group all had nosocomial infection and none had a pathogen detected.

2. Sepsis with lactic acidosis, this group had the causative pathogen detected in all patients, of those the vast majority (92\%) was bacteremic. In addition, this group had the highest frequency of organ dysfunction at admission to the ICU.

3. Community-acquired, sterile sepsis, these patients often suffered from a respiratory tract infection or an abdominal infection.

4. Abdominal sepsis with pathogen detected, the infection was community-acquired.

5. Nosocomial, pathogen-detected sepsis.

6. Sepsis with gram-positive bacteria, the infections were community-acquired, often in the respiratory tract or skin and soft tissue infections.

7. Imunosuppressed sepsis, these patients had antibiotic therapy prior to microbial sampling and were yet bacteremic.

8. Urinary tract infection sepsis, the pathogen, Enterobacterales, was always detected.

90-day mortality for the different classes was compared in a Kaplan-Meier model, Fig. 3.

There was a difference in 90-days mortality between classes $(p<0.01)$ with the lowest mortality in class 8 , Urinary tract infection sepsis and the highest in class 2, Sepsis with lactic acidosis.

\section{Discussion}

The main findings in this study are that firstly, bacteremia is associated with poor outcome, and secondly that a higher percentage than previously reported of ICU patients with sepsis had positive blood cultures and other microbiological samples when analyzed with clinical chart reviewed sepsis diagnosis. Thirdly, blood culture positivity is affected by prior antibiotic treatment.

The high proportion of bacteremic patients, $54 \%$ in this study compared to $7-37 \%$ in other studies, may at least partially be explained by the manual chart review in the present study $(2,3,6,8,11-13)$. Two prior studies classify patients as bacteremic depending on ICD-code, which might lead to a proportion of patients being misclassified as culture-negative $(9,11)$. Others define sepsis as blood cultures drawn in combination with, for example two Systemic Inflammatory Response Syndrome (SIRS) criteria or ICU care, which includes other diagnoses than sepsis as well (11-13).

We applied strict inclusion criteria of patients with at least blood cultures drawn and fulfilling an infection diagnosis and a corresponding sepsis-3 diagnosis. If we would have relied on administrative data like ICD-codes or electronic health record algorithms based on blood cultures for diagnosis, 95 (15\%) patients not fulfilling infection or sepsis-3 definitions would have been included. Further, ICD-based strategies would even risk to include the 140 (18\%) patients without blood cultures taken. As a poor accuracy of ICD-coding for sepsis is 
well documented, clinical chart reviews should be considered "gold standard" in sepsis epidemiology studies (20-23). Results from studies based on automated electronic health record data, should be interpreted with caution even if based on large amounts of data.

Another explanation for the high proportion of bacteremic sepsis might be the high morbidity in this cohort, since bacteremic patients had even higher severity of illness and higher mortality than their non-bacteremic counterparts. The sterile sepsis proportion is similar to the numbers described in a previous prospective study on patients with septic shock where 2651 patients (31\%) had sterile sepsis (10). We also included other microbiological samples than culture, although they constituted only a small proportion of the pathogen-detected nonbacteremic sepsis group.

Sepsis is a highly heterogenous condition and different foci of infection have both different mortalities as well as different diagnostic yield of cultures and other microbiological analyses. In the present study propensity score match was used to reduce baseline differences between the groups and to estimate differences in morbidity and mortality with minimal bias.

Bacteremic patients demonstrated higher mortality rate than controls in the present study.

Previous studies have resulted in evidence both for and against bacteremia being associated with higher mortality $(6,24-28)$. In a large, prospective study by Phua et a/mortality was not higher among culture-positive patients in a multivariate analysis and Gupta et al found higher mortality in culture-negatives, however antibiotic therapy preceding culture sampling was not included in the models $(2,9)$. Nannan Panday et al took prior antibiotic therapy into account when retrospectively analyzing a prospectively gathered cohort, and found higher mortality among bacteremic patients (6). We demonstrate that preceding antibiotic therapy is a confounder affecting mortality in nonbacteremic patients. Possibly, a proportion of non-bacteremic sepsis might be bacteremic but without growth in the blood cultures due to antibiotic therapy preceding culture sampling.

Higher mortality in bacteremic sepsis and in non-bacteremic sepsis with prior antibiotic treatment can also be indicative of bacterial load in blood being associated with sepsis severity, which previously has been demonstrated for isolated pathogens (29-32).

Still, $30 \%$ of the patients had sterile sepsis, i.e. negative in all microbiology samples, with a mortality of $44 \%$. With the high incidence of sepsis and the emerging antimicrobial resistance, sterile sepsis is a substantial cause of morbidity which needs to be examined further. Sterile sepsis has been speculated to depend on misdiagnosis of other conditions, preceding antibiotic therapy, insufficient culture sampling, handling or culture techniques (6). The clinical chart review minimizes the misdiagnosis of other conditions and Kethireddy et al, who found similar mortalities in culture-positive and culture-negative patients with septic shock, demonstrated an increase in mortality with delayed antimicrobial therapy in both groups, indicating bacterial cause (10).

Our results suggest sterile and non-bacteremic sepsis to partially depend on prior antibiotic therapy. The proportion of sterile sepsis patients decreased from $43 \%$ with prior antibiotic therapy to $22 \%$ sterile sepsis patients without prior antibiotic therapy, and there was a similar decrease from $63 \%$ bacteremic patients without prior antibiotic therapy to $37 \%$ bacteremic sepsis patients with prior antibiotic therapy. Thus, antibiotic therapy seems to be a predictor for culture-negative sepsis. This is in contrast to Previsdomini et al, although they also noticed this trend but was possibly limited by a smaller sample size (13).

The LCA offers clinical subphenotypes in a classification, that might not be identified and its impact might not be tested assuming accepted standards. Neither the site nor the microbiology alone distinguished the classes, yet the combinations together with where the infection was acquired, immunosuppression and lactic acidosis were important for class distinction but also for outcome. This finding is in accordance with the propensity score analysis. When defining subphenotypes in sepsis, pathogen detection in microbial samples seems to have a high impact on probability of belonging to a class. This has previously been demonstrated for septic shock, while it was not a variable in a LCA used for ARDS and might have an impact in staging models like the PIRO system (predisposition, insult, response, organ dysfunction) (14, $15,33)$.

The strengths of this study are the considering of the effect of antibiotic therapy prior to collection of microbiological samples, the inclusion of other microbial samples than cultures (e.g. PCR) and the data on and the high number of microbial samples collected. Further, the results do not solely rely on administrative or microbial data. All infection diagnoses and all data from microbial analysis have been reviewed by an infectious disease specialist. The proportion sterile sepsis might be underestimated since patients without an identified pathogen are less likely to obtain an infection diagnosis.

The major weakness of this study is the retrospective design. As microbiological samples were ordered as part of clinical workup, insufficient culture sampling might contribute to the microbiology negative cohorts. Fewer samples were drawn from blood, urine and wounds for microbiology-negative patients and fewer respiratory tract samples were withdrawn from bacteremic patients, although when

Page 14/19 
cultures were compared to presumed infectious foci, there were no significant differences in ratio. Also, the handling of microbiological samples and laboratory techniques were part of clinical practice and out of study control. Other weaknesses of the study are the relatively small size and the single center conduct. The classes created by the LCA were created out of, and limited to observable characteristics of the variables entered.

\section{Conclusions}

In summary, bacteremia as well as preceding antibiotic treatment in non-bacteremic patients are related to poor outcome. Bacteremia is more common than previously described in sepsis, when a clinical chart review is used as gold-standard. A substantial portion of sepsis patients that remain microbiology-negative cannot be attributed to misdiagnosis or preceding antibiotic treatment. For reducing population heterogeneity and improve the outcome of trials and treatment for sepsis, microbiological-negativity is an important factor of a subphenotype.

\section{Abbreviations}

ABIC Adjusted Bayesian Information Criterion

AIC Akaike Information Criterion

BIC Bayesian Information Criterion (BIC),

Cl Confidence Interval

COPD Chronic Obstructive Pulmonary Disease

ICD International Classification of Disease

ICU Intensive Care Unit

IQR Interquartile Range

LCA Latent Class Analysis

PCR Polymerase Chain Reaction

PIRO Predisposition, Insult, Response, Organ dysfunction

SOFA Sequential Organ Failure Assessment

\section{Declarations}

\section{Ethics approval and consent to participate}

The study was approved by the Regional Ethical Review Boar, decision number 2015/285. The Regional Ethics Review Board waived the requirement for informed consent.

Consent for publication Not applicable

Availability of data and material Anonymised data from the study is available upon reasonable request.

\section{Competing interests}

All authors have declared no relevant conflicts of interest.

\section{Funding}

Swedish Government Funds for Clinical Research (ALF), Region Skåne, the Crafoord foundation, the foundation of Alfred Österlund are acknowledged for generous support.

\section{Authors' contributions}


LM and AL had full access to all of the data in the study and takes responsibility for the integrity of the data and the accuracy of the data analysis.

Study concept and design: AL, LM

Acquisition, analysis, or interpretation of data: All authors

Drafting of the manuscript: $\mathrm{LM}, \mathrm{AL}, \mathrm{FK}, \mathrm{BC}$

Critical revision of the manuscript for important intellectual content: All authors

Statistical analysis: LM, FK

\section{Acknowledgements}

\section{References}

1. Singer M, Deutschman CS, Seymour CW, Shankar-Hari M, Annane D, Bauer M, et al. The Third International Consensus Definitions for Sepsis and Septic Shock (Sepsis-3). Jama. 2016;315(8):801-10.

2. Phua J, Ngerng W, See K, Tay C, Kiong T, Lim H, et al. Characteristics and outcomes of culture-negative versus culture-positive severe sepsis. Crit Care (London England). 2013;17(5):R202.

3. Rhee C, Dantes R, Epstein L, Murphy DJ, Seymour CW, Iwashyna TJ, et al. Incidence and Trends of Sepsis in US Hospitals Using Clinical vs Claims Data, 2009-2014. Jama. 2017;318(13):1241-9.

4. Mellhammar L, Wullt S, Lindberg A, Lanbeck P, Christensson B, Linder A. Sepsis Incidence: A Population-Based Study. Open forum infectious diseases. 2016;3(4):ofw207.

5. Todorovic Markovic M, Pedersen C, Gottfredsson M, Todorovic Mitic M, Gaini S. Epidemiology of community-acquired sepsis in the Faroe Islands - a prospective observational study. Infectious diseases (London England). 2019;51(1):38-49.

6. Nannan Panday RS, Lammers EMJ, Alam N, Nanayakkara PWB. An overview of positive cultures and clinical outcomes in septic patients: a sub-analysis of the Prehospital Antibiotics Against Sepsis (PHANTASi) trial. Critical care (London, England). $2019 ; 23(1): 182$.

7. Ljungstrom L, Andersson R, Jacobsson G. Incidences of community onset severe sepsis, Sepsis-3 sepsis, and bacteremia in Sweden - A prospective population-based study. PloS one. 2019;14(12):e0225700.

8. Vincent JL, Sakr Y, Sprung CL, Ranieri VM, Reinhart K, Gerlach H, et al. Sepsis in European intensive care units: results of the SOAP study. Crit Care Med. 2006;34(2):344-53.

9. Gupta S, Sakhuja A, Kumar G, McGrath E, Nanchal RS, Kashani KB. Culture-Negative Severe Sepsis: Nationwide Trends and Outcomes. Chest. 2016;150(6):1251-9.

10. Kethireddy S, Bilgili B, Sees A, Kirchner HL, Ofoma UR, Light RB, et al. Culture-Negative Septic Shock Compared With Culture-Positive Septic Shock: A Retrospective Cohort Study. Crit Care Med. 2018;46(4):506-12.

11. Armstrong-Briley D, Hozhabri NS, Armstrong K, Puthottile J, Benavides R, Beal S. Comparison of length of stay and outcomes of patients with positive versus negative blood culture results. Proceedings (Baylor University Medical Center). 2015;28(1):10 - 3.

12. Sigakis MJG, Jewell E, Maile MD, Cinti SK, Bateman BT, Engoren M. Culture-Negative and Culture-Positive Sepsis: A Comparison of Characteristics and Outcomes. Anesthesia analgesia. 2019;129(5):1300-9.

13. Previsdomini M, Gini M, Cerutti B, Dolina M, Perren A. Predictors of positive blood cultures in critically ill patients: a retrospective evaluation. Croatian Med J. 2012;53(1):30-9.

14. Calfee CS, Delucchi K, Parsons PE, Thompson BT, Ware LB, Matthay MA. Subphenotypes in acute respiratory distress syndrome: latent class analysis of data from two randomised controlled trials. The Lancet Respiratory medicine. 2014;2(8):611-20.

15. Gardlund B, Dmitrieva NO, Pieper CF, Finfer S, Marshall JC, Taylor Thompson B. Six subphenotypes in septic shock: Latent class analysis of the PROWESS Shock study. J Crit Care. 2018;47:70-9.

16. Seymour CW, Kennedy JN, Wang S, Chang CH, Elliott CF, Xu Z, et al. Derivation, Validation, and Potential Treatment Implications of Novel Clinical Phenotypes for Sepsis. Jama. 2019;321(20):2003-17.

17. Calandra T, Cohen J. The international sepsis forum consensus conference on definitions of infection in the intensive care unit. Crit Care Med. 2005;33(7):1538-48. 
18. Leligdowicz A, Dodek PM, Norena M, Wong H, Kumar A, Kumar A. Association between source of infection and hospital mortality in patients who have septic shock. Am J Respir Crit Care Med. 2014;189(10):1204-13.

19. Shankar-Hari M, Harrison DA, Rowan KM. Differences in Impact of Definitional Elements on Mortality Precludes International Comparisons of Sepsis Epidemiology-A Cohort Study Illustrating the Need for Standardized Reporting. Crit Care Med. 2016;44(12):2223-30.

20. Gaieski DF, Edwards JM, Kallan MJ, Carr BG. Benchmarking the incidence and mortality of severe sepsis in the United States. Crit Care Med. 2013;41(5):1167-74.

21. Wilhelms SB, Walther SM, Huss F, Sjoberg F. Severe sepsis in the ICU is often missing in hospital discharge codes. Acta anaesthesiologica Scandinavica. 2017;61(2):186-93.

22. Wilhelms SB, Huss FR, Granath G, Sjoberg F. Assessment of incidence of severe sepsis in Sweden using different ways of abstracting International Classification of Diseases codes: difficulties with methods and interpretation of results. Crit Care Med. 2010;38(6):14429.

23. Johansson D, Ekstrom H, Beronius E, Rasmussen M. [Systematic medical record review in Skane. Diagnostic codes were often wrong in severe sepsis and septic shock]. Lakartidningen. 2015;112.

24. Zahar JR, Timsit JF, Garrouste-Orgeas M, Francais A, Vesin A, Descorps-Declere A, et al. Outcomes in severe sepsis and patients with septic shock: pathogen species and infection sites are not associated with mortality. Crit Care Med. 2011;39(8):1886-95.

25. Artero A, Inglada L, Gomez-Belda A, Capdevila JA, Diez LF, Arca A, et al. The clinical impact of bacteremia on outcomes in elderly patients with pyelonephritis or urinary sepsis: A prospective multicenter study. PloS one. 2018;13(1):e0191066.

26. Hsu CY, Fang HC, Chou KJ, Chen CL, Lee PT, Chung HM. The clinical impact of bacteremia in complicated acute pyelonephritis. Am J Med Sci. 2006;332(4):175-80.

27. Brooks D, Smith A, Young D, Fulton R, Booth MG. Mortality in intensive care: The impact of bacteremia and the utility of systemic inflammatory response syndrome. Am J Infect Control. 2016;44(11):1291-5.

28. Laupland KB, Davies HD, Church DL, Louie TJ, Dool JS, Zygun DA, et al. Bloodstream infection-associated sepsis and septic shock in critically ill adults: a population-based study. Infection. 2004;32(2):59-64.

29. Ziegler I, Cajander S, Rasmussen G, Ennefors T, Molling P, Stralin K. High nuc DNA load in whole blood is associated with sepsis, mortality and immune dysregulation in Staphylococcus aureus bacteraemia. Infectious diseases (London, England). 2019;51(3):21626.

30. Rello J, Lisboa T, Lujan M, Gallego M, Kee C, Kay I, et al. Severity of pneumococcal pneumonia associated with genomic bacterial load. Chest. 2009;136(3):832-40.

31. Hackett SJ, Guiver M, Marsh J, Sills JA, Thomson AP, Kaczmarski EB, et al. Meningococcal bacterial DNA load at presentation correlates with disease severity. Arch Dis Child. 2002;86(1):44-6.

32. Marra AR, Edmond MB, Forbes BA, Wenzel RP, Bearman GM. Time to blood culture positivity as a predictor of clinical outcome of Staphylococcus aureus bloodstream infection. J Clin Microbiol. 2006;44(4):1342-6.

33. Levy MM, Fink MP, Marshall JC, Abraham E, Angus D, Cook D, et al. 2001 SCCM/ESICM/ACCP/ATS/SIS International Sepsis Definitions Conference. Intensive care medicine. 2003;29(4):530-8.

\section{Figures}




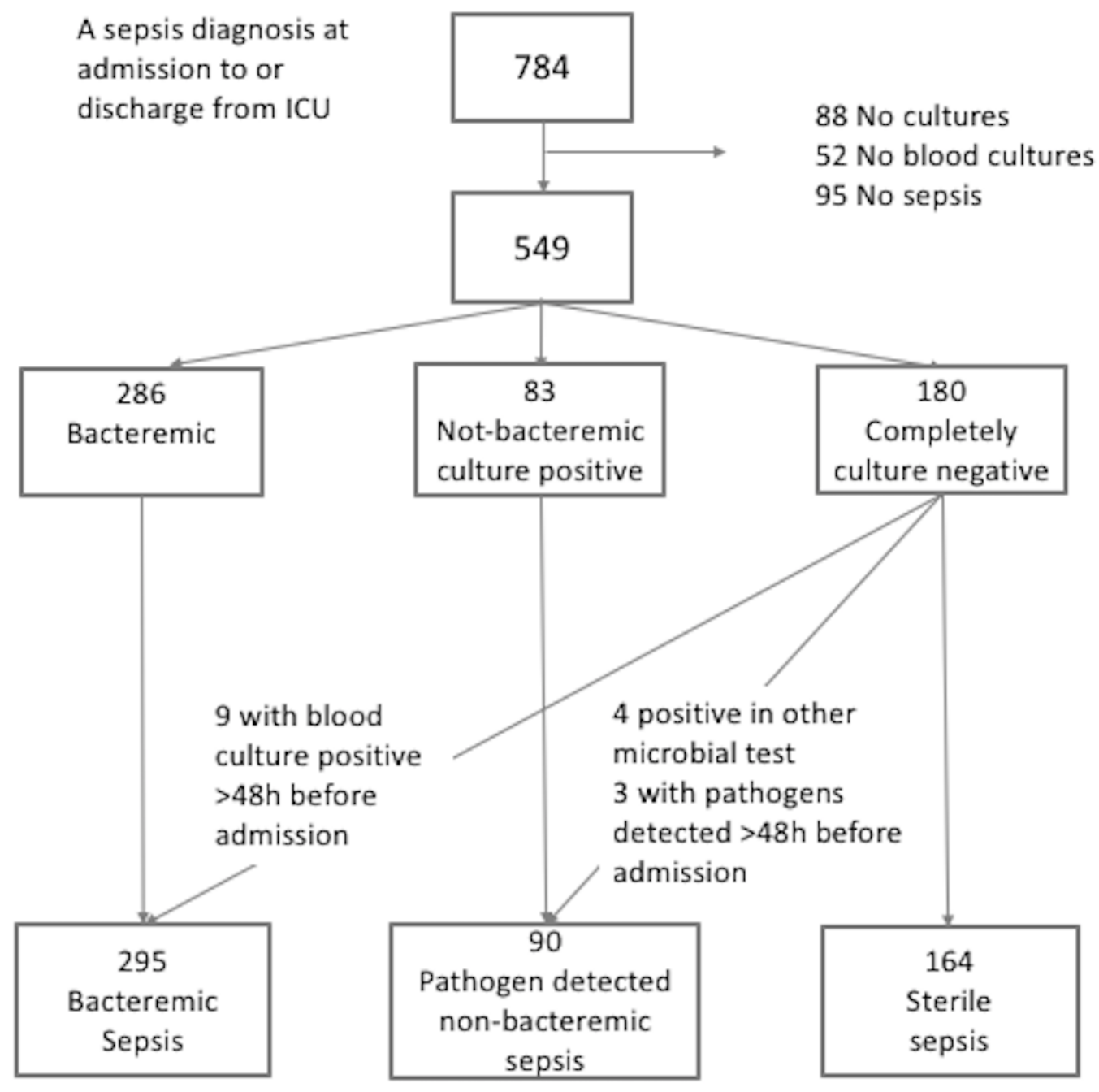

Figure 1

Flow chart
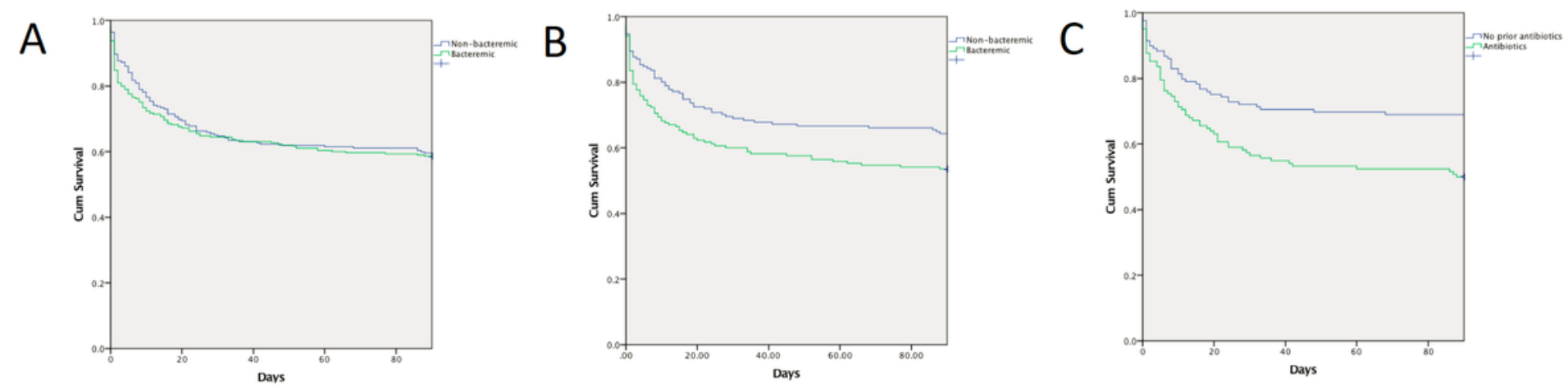

Figure 2

Mortality in bacteremic and non-bacteremic patients. 90-day mortality in bacteremic and non-bacteremic patients, non-adjusted cohort, showing no significant difference $42 \%$ vs $41 \%, p=0.74$. b. Mortality in bacteremic and non-bacteremic patients, propensity matched cohorts. 90-day mortality in bacteremic and non-bacteremic patients, propensity matched cohorts, showing a significant difference $47 \%$ vs $36 \%$ 
$\mathrm{p}=0.04 \mathrm{c}$. Mortality in non-bacteremic patients with/without prior antibiotic therapy. 90-day mortality in non-bacteremic patients with/without prior antibiotic therapy showing a significant difference $51 \%$ vs $31 \%, p<0.01$.

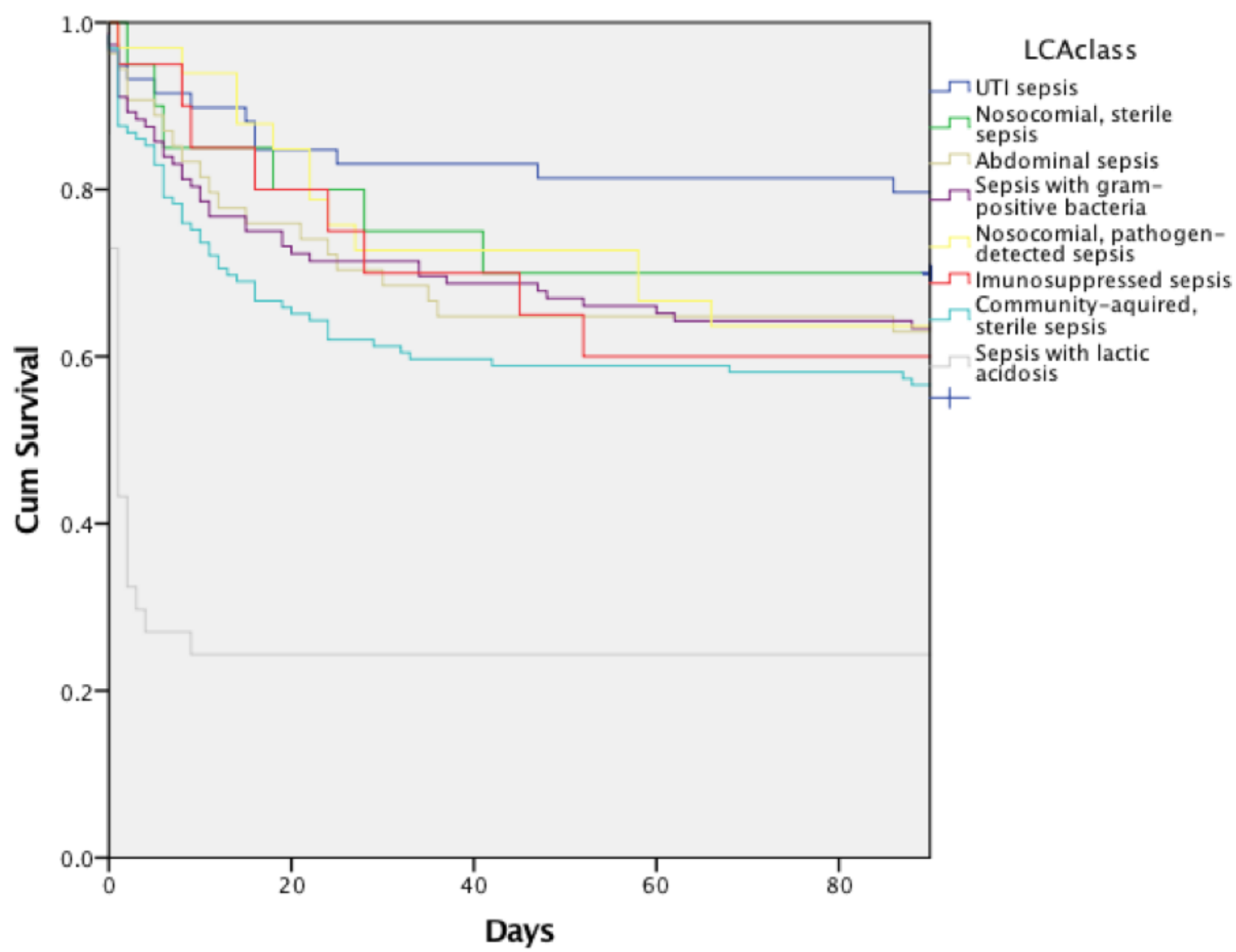

\section{Figure 3}

Mortality in the eight classes derived from LCA 90-day mortality in the eight classes derived from LCA, $p<0.01$ 